\title{
Texture Classification in Lung CT Using Local Binary Patterns
}

\author{
Lauge Sørensen ${ }^{1}$, Saher B. Shaker ${ }^{2}$, and Marleen de Bruijne ${ }^{1,3}$ \\ ${ }^{1}$ Department of Computer Science, University of Copenhagen, Denmark, \\ \{lauges, marleen\}@diku.dk \\ ${ }^{2}$ Department of Cardiology and Respiratory Medicine, Hvidovre University Hospital, \\ Copenhagen, Denmark \\ ${ }^{3}$ Biomedical Imaging Group Rotterdam, Erasmus MC, The Netherlands
}

\begin{abstract}
In this paper we propose to use local binary patterns (LBP) as features in a classification framework for classifying different texture patterns in lung computed tomography. Image intensity is included by means of the joint LBP and intensity histogram, and classification is performed using the $k$ nearest neighbor classifier with histogram similarity as distance measure.

The proposed method is evaluated on a set of 168 regions of interest comprising normal tissue and different emphysema patterns, and compared to a filter bank based on Gaussian derivatives. The joint LBP and intensity histogram, achieving a classification accuracy of $95.2 \%$, shows superior performance to using the common approach of taking moments of the filter response histograms as features, and slightly better performance than using the full filter response histograms instead. Classification results are better than some of those previously reported in the literature.
\end{abstract}

\section{Introduction}

Chronic obstructive pulmonary disease (COPD) is a major cause of death and a growing health problem worldwide. In the United States it is the fourth leading cause of morbidity and mortality and it is estimated to be ranked the fifth most burdening disease worldwide by 2020 [1]. COPD is a chronic lung disease characterized by limitation of airflow in the airway and it comprises two components: Chronic bronchitis, which is an inflammation of the small airways, and emphysema, which is characterized by gradual loss of lung tissue.

The primary diagnostic tools for COPD are the lung function tests (LFT). However, LFT has a low sensitivity and is not capable of detecting early stages of COPD. Another diagnostic tool that is gaining more and more attention is computed tomography (CT) imaging. CT is a sensitive method for diagnosing emphysema and both visual and quantitative CT are closely correlated with the pathological extent of emphysema 2]. This makes CT suitable for both early detection and study of COPD as well as to monitor effect of different treatments.

We focus on the assessment of emphysema, which is thought to be the main cause of shortness of breath and disability in COPD. In CT emphysema lesions,

D. Metaxas et al. (Eds.): MICCAI 2008, Part I, LNCS 5241, pp. 934 941, 2008.

(C) Springer-Verlag Berlin Heidelberg 2008 

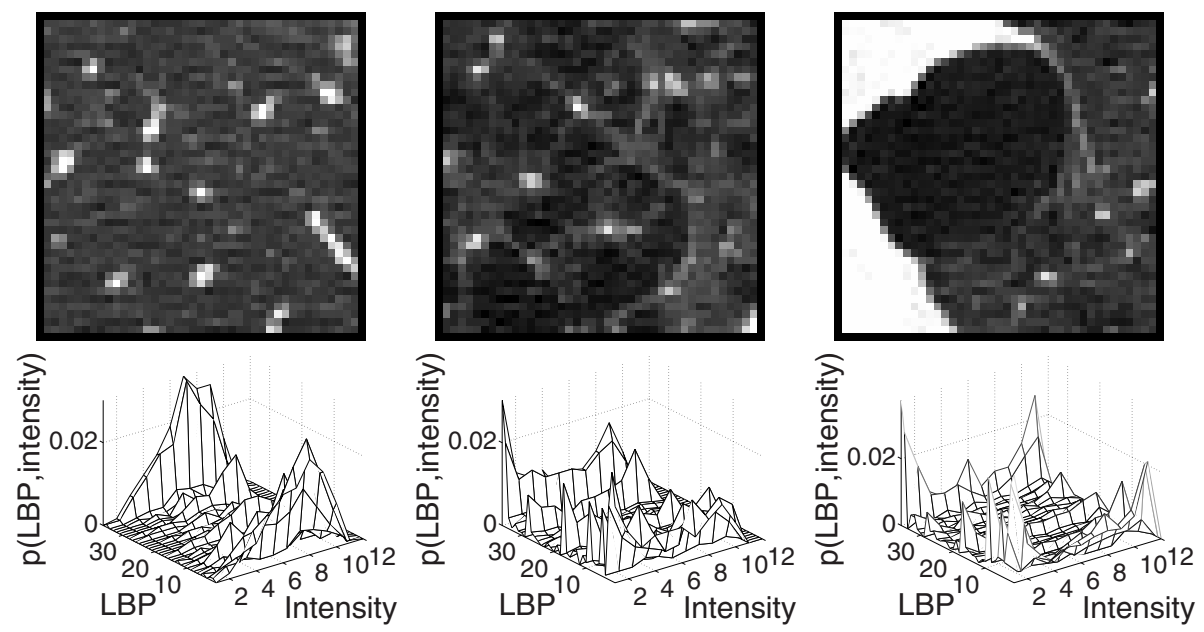

Fig. 1. Example ROIs and corresponding joint LBP and intensity histograms. Top row: $41 \times 41$ pixel ROIs showing examples of the appearance of the three classes used in the experiments. Top Left: Normal tissue. Top middle: Centrilobular emphysema Top right: Paraseptal emphysema. Bottom row: Joint histogram between LBP $(R=$ 1 and $P=8)$ and intensity in the center pixel corresponding to the example ROIs in the top row.

or bullae, are visible as areas of abnormally low attenuation values, close to that of air. Different objective parameters for quantification of emphysema can be derived from the histogram of $\mathrm{CT}$ attenuation values, and the most common one is the relative area of emphysema 3. Usually emphysema is classified into three subtypes and we will adopt the naming and definitions from Webb et al. 4. These subtypes are: Centrilobular emphysema (CLE) defined as multiple, small, spotty lucencies, that may have thin walls, paraseptal emphysema (PSE) defined as multiple, lucencies in a single layer along the pleura, commonly with thin walls visible, and panlobular emphysema (PLE) defined as a lucent lung with small pulmonary vessels. The middle and right column of the top row of Figure 1 show examples of CLE and PSE respectively. Common for the quantitative methods based on the attenuation histogram are that they ignore the possibly valuable information inherent in the emphysema disease patterns, such as subtype, shape, and size distribution. This was exemplified in a recent clinical study that reported discrepancies between disease pattern based visual scoring and relative area of emphysema for assessing the craniocaudal distribution of emphysema in the three subtypes [5].

One way to objectively analyze the properties of the disease patterns is to use texture analysis [6]. This can be turned into a quantitative measure by performing pixel classification of each pixel in the lung based on textural appearance in a local region around the pixels. Several publications exist on classifying emphysema and other disease patterns in lung CT images using texture features. 
[7/819 uses measures on co-occurrence matrices, measures on run-length matrices, moments of the attenuation or intensity histogram, and in some cases fractal dimension as features. Sluimer et al. [10] used a filter bank of Gaussians and Gaussian derivatives.

In this paper we propose to use local binary patterns (LBP), formulated by Ojala et al. [11, as texture features in a pattern classification system for discriminating between normal tissue (NT), CLE, and PSE. Using LBP it is possible to unify structural and statistical information by histograms of microstructures, and LBP are simple to compute. LBP have shown promising results in various applications in computer vision, and have been applied in other problems in medical imaging, for example resulting in a reduction of the number of false positives in mammographic mass detection [12. To our knowledge LBP have not been applied to lung texture classification before. LBP is by design invariant to intensity, thus we also propose to include the intensity distribution. Previous work on lung texture classification has used moments of the intensity histogram as features [7 8910]. We propose to instead use the full intensity histogram, together with LBP, by means of the joint LBP and intensity histogram, and to use it in a $k$ nearest neighbor $(k \mathrm{NN})$ classifier with histograms similarity as distance measure. The proposed method is compared to that of a filter bank based on Gaussian derivatives.

\section{Methods}

In the following the classification system is described. Section 2.1] describes the LBP features we use for characterizing the lung texture, Section 2.2 presents an alternative group of features used for comparison purposes, and Section 2.3 describes the classification framework.

\subsection{Local Binary Patterns}

The features that we use are based on the local binary patterns (LBP) proposed by Ojala et al. [11]. In general, LBP measures the local structure at a given pixel using $P$ samples on a circle of radius $R$ around the pixel and summarizes this information with a unique code for each local structure or pattern. The operator is highly non-linear and detects microstructures in the image at different resolutions governed by the parameter $R$, for example spots, edges, corners, etc., exemplified in the right part of Figure 2. The basic steps for calculating the LBP code in a pixel position in a given image are illustrated in the left part of Figure2. Applying the LBP operator to an image results in a LBP code image. Based on this an LBP histogram is formed. Note that no quantization is needed and that the LBP codes are directly accumulated into a histogram. The LBP histogram combines structural and statistical approaches, LBP detects microstructures in the image, and the histogram captures the distribution of these microstructures. We use the rotation invariant formulation of LBP which is accomplished as illustrated in Figure 2 (c), see [11] for more details. 


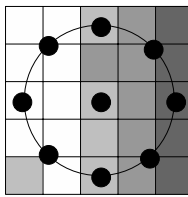

(a)

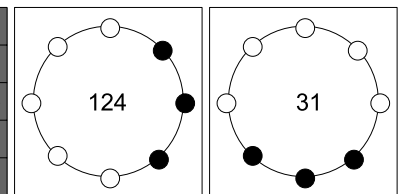

(b)

(c)

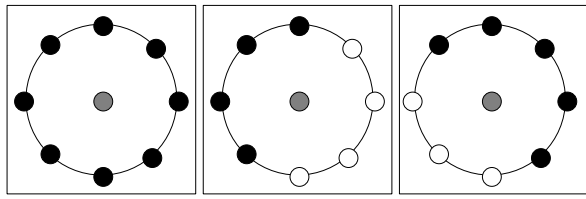

Spot

Edge

Corner

Fig. 2. Illustration of LBP. Left: The basic steps in computing the LBP code for a given pixel position: (a) the operator is centered on the given pixel and $P$ equidistant samples are taken on the circle of radius $R$ around the center; (b) the obtained samples are turned into 0's and 1's by applying a sign function with the center pixel value as threshold. By choosing a fixed sample position on the circle as the leading bit, the samples can be turned into a binary number. Thus each pattern has an associated unique binary number; (c) rotation invariance is achieved by bitwise shifting the binary pattern clock-wise until the lowest binary number is found. Right: Some of the microstructures that LBP are measuring.

LBP are invariant to any monotonic gray-scale transformation of the image. This is however not a desired property when dealing with CT images, where values are measurements of a physical property of the tissue displayed. [11] describes how to include contrast into LBP by making a joint histogram between the LBP and the variance of the intensities in the LBP samples. In a similar fashion we include the distribution of the intensities in the center pixels, by forming the joint histogram between the LBP and the intensities in the center pixels. This results in a histogram containing information about how the LBP varies at different intensity ranges, which we expect to be valuable information. The bottom row of Figure 1 contain examples of joint LBP and intensity histograms computed from the ROIs shown in the top row.

\subsection{A Filter Bank Based on the Gaussian Function and Its Derivatives}

For comparison we introduce a second group of features, which are based on the Gaussian function $G(\mathbf{x} ; \sigma)$ and combinations of derivatives of the Gaussian function. By varying the standard deviation $\sigma$ of the function in a discrete manner we obtain a whole bank of filters that can be applied to the images. Using a filter bank of various derivatives of the Gaussian function for lung texture classification was proposed by Sluimer et al. [10], where the Gaussian function itself was also included to make the filter bank sensitive to offsets in absolute intensity. The filters that we use are similar to those used in [10], except that the filters we use are all rotation invariant. The filter bank comprise the following filters: $G(\mathbf{x} ; \sigma)$; the Laplacian of the Gaussian $\nabla^{2} G(\mathbf{x} ; \sigma)$; the gradient magnitude $|\nabla G(\mathbf{x} ; \sigma)|_{2} ;$ the Gaussian curvature $K(\mathbf{x} ; \sigma)=\partial^{2} G(\mathbf{x}, \sigma) / \partial x^{2}+\partial^{2} G(\mathbf{x}, \sigma) / \partial y^{2}-$ $2 \partial^{2} G(\mathbf{x}, \sigma) / \partial x y$. 


\subsection{Classification Framework}

Classification is done using the $k \mathrm{NN}$ classifier 13. When using the filter bank features, of Section 2.2, feature selection is applied using the sequential forward selection algorithm [13] for deciding which filters at which scales to use. When using the LBP features, of Section 2.1, several combinations of radii for multiresolution analysis are evaluated.

In [7/8]9|10] intensity and filter response distributions were represented by up to four moments of the histogram. This way a more compact representation is obtained at the expense of information loss. We use the full histogram instead and apply histogram similarity as distance measure in the $k \mathrm{NN}$ classifier. The histograms of the intensities and the filter responses are constructed using nonlinear binning, where the binning is found by employing two rules on the total distribution of the ROIs in the training set: The distribution of the total distribution should be uniform and the number of bins is $\lfloor\sqrt[3]{N}\rfloor$, where $N$ is the number of pixels in the ROI. Histogram intersection is used as the similarity measure between histogram $H$ and histogram $K$

$$
L_{\text {hist }}(H, K)=1-\sum_{b=1}^{B} \min \left(H_{b}, K_{b}\right),
$$

where $H_{b}$ denotes bin $b$ of histogram $H, B$ is the number of histogram bins and the histograms are assumed normalized to sum to one. Other histogram similarity measures could be used, for example the G statistic [11] or the earth movers distance [14. In the case of measuring combined histogram similarity of $N$ different histograms, we sum the individual histogram similarities computed using (1)

$$
L_{N}=\sum_{n=1}^{N} L_{h i s t}\left(H^{n}, K^{n}\right) .
$$

\section{$3 \quad$ Experiments and Results}

The data used for the experiments is collected from a set of thin-slice CT images of the thorax. CT was performed using GE equipment (LightSpeed QX/i; GE Medical Systems, Milwaukee, WI, USA) with four detector rows, using the following parameters: 1.25 - $\mathrm{mm}$ collimation, tube voltage $140 \mathrm{kV}$ and tube current 200 milliampere $(\mathrm{mA})$. The slices were reconstructed using a high spatial resolution (bone) algorithm. A population of 25 individuals, 8 healthy non-smokers, 4 smokers without COPD, and 13 smokers diagnosed with moderate or severe COPD according to LFT [1] were scanned in the upper, middle, and lower lung, resulting in a total of $75 \mathrm{CT}$ slices. Visual assessment of the leading pattern, either NT, CLE, PSE, or PLE, in each of these slices was done individually by an experienced chest radiologist and a CT experienced pulmonologist. In $47 \%$ of the slices there was disagreement and in the slices where both human observers agreed on an emphysematous leading pattern, there was disagreement on the 
Table 1. Left: Evaluation of the six different lung texture classification approaches. Right: Confusion matrices for the six approaches, in which the rows define the true class and the columns the estimated class. The top left matrix is for FB1, the top middle FB2, etc.

\begin{tabular}{|l|c|c|c|ccc|ccc|ccc|}
\hline Approach & Accuracy $(\%)$ & $\boldsymbol{p}$ & & NT CLE PSE & NT & CLE PSE & NT CLE PSE \\
\hline FB1 & 61.3 & $\approx 0$ & NT & 17 & 27 & 15 & 55 & 0 & 4 & 41 & 8 & 10 \\
FB2 & 94.0 & 0.724 & CLE & 19 & 31 & 0 & 2 & 48 & 0 & 6 & 42 & 2 \\
LBP1 & 79.2 & $\approx 0$ & PSE & 4 & 0 & 55 & 4 & 0 & 55 & 3 & 6 & 50 \\
INT & 87.5 & 0.004 & NT & 51 & 3 & 5 & 56 & 1 & 2 & 55 & 0 & 4 \\
LBP2 & 92.3 & 0.228 & CLE & 2 & 48 & 0 & 1 & 49 & 0 & 1 & 49 & 0 \\
LBP3 & 95.2 & - & PSE & 8 & 3 & 48 & 8 & 1 & 50 & 2 & 1 & 56 \\
\hline
\end{tabular}

emphysema class in $40 \%$ of the cases. Consensus readings were obtained in all cases of disagreement. 168 non-overlapping ROIs were annotated in the slices representing the three classes: NT (59 observations from non-smokers), CLE (50 observations), and PSE (59 observations). The NT ROIs were annotated in the non-smokers and the CLE and PSE ROIs were annotated in the two smokers patient classes, within the area(s) of the leading pattern. PLE was excluded due to underrepresentation in the data set (only 2 out of 20 individuals diagnosed with COPD).

Six different approaches are evaluated and compared:

- A filter bank with the first four moments of filter responses, standardized to unit variance, as features. In this approach we use Euclidean distances in a feature space in the $k \mathrm{NN}$ classifier $(\mathrm{FB} 1)$.

- A filter bank with histograms of filter responses, combined using (2), as features (FB2).

- Basic rotation invariant LBP histograms (LBP1).

- Intensity histograms (INT).

- LBP histograms and intensity histograms, combined using (2) (LBP2).

- Joint LBP and intensity histograms (LBP3).

The six different approaches are each evaluated using leave-one-out on patient level, thus in each trial all ROIs from one patient are held out and used for testing. The remaining ROIs are split into a training set and validation set using a $50 \% / 50 \%$ split on patient level on each class, such that all ROIs from half the patients of each class are put in the training set and the rest in the validation set. Using the training set and validation set the model is trained, meaning that the optimal setting for the parameters listed in the next paragraph are selected. Subsequently, the held out ROIs are classified with the selected parameter setting using all the samples in the training set and validation set in the $k \mathrm{NN}$.

The following parameter values are considered during training. In the Gaussian based filter bank the following scales are used for all filters $\sigma=[0.5,1,2,4,8]$ pixels. In the LBP the following radii are used $R=[1,2]$ pixels with corresponding number of samples $P=[8,16]$. Single radii as well as a combination of the two are tried during training. Combining operators results in a multiresolution 
analysis, and the combination is done using (2). Common parameters for all six approaches are: ROI size $=[31 \times 31,41 \times 41,51 \times 51]$ pixels and number of neighbors in the $k \mathrm{NN}$ classifier $k=[1,2, \ldots, 10]$ neighbors.

The performance of the six approaches is summarized in Table1left, where the best performance (95.2\% classification accuracy) is achieved by LBP3. The table also includes $p$-values found using McNemar's test [15], comparing our proposed approach, LBP3, against the other approaches. Further, confusion matrices are shown in Table 1 right.

\section{Discussion and Conclusion}

LBP3 performs best achieving a classification accuracy of $95.2 \%$, however, it is not significantly different $(p=0.72)$ from the second best approach, FB2 which achieves an accuracy of $94.0 \%$. There is an indication that there is a gain in modeling the joint LBP and intensity histogram instead of using the individual LBP and intensity histogram together, which is evident from the fact that LBP2 has a lower performance $(92.3 \%)$, but this difference is not significant $(p=0.23)$. We expect that a larger data set would make the importance of joint modeling more evident, but LBP2 may remain a viable, computationally cheaper, alternative. LBP alone achieves a decent performance $(79.2 \%)$ but including intensity information improves the performance considerably as expected; LBP3 is significantly better than LBP1 $(p \approx 0)$. That the intensity information is important is also evident from the fact that INT achieves better performance than LBP1. In this setting, moments of the histogram clearly provide a too simple representation of the full distribution; the performance of FB1 is the lowest of all (61.3\%). This is an interesting finding that should be investigated further, since most previous work on texture classification in lung CT uses moments, for example [7/8910].

Comparing our results to some of the previous work, the performance of LBP3 is actually very good. 8 reports an overall sensitivity of $60.3 \%$ and an overall specificity of $86.7 \%$, where for LBP3 the sensitivity is $97.3 \%$ and the specificity is $93.2 \%$ (when comparing NT versus CLE and PSE). 10 reports a classification accuracy of $76 \%$ when using a $k \mathrm{NN}$ classifier, whereas the classification accuracy of LBP3 is $95.2 \%$. Off course the results are not directly comparable, due to differences in the data, the choice of classes, etc., but this comparison at least gives an indication of the usefulness of our proposed approach.

The relatively high level of disagreement between the two human expert observers on determining the leading pattern, illustrates the importance and usefulness of developing objective methods for characterizing emphysema patterns. These methods should be based on consensus readings of as many experts as possible, or be unsupervised.

In this paper we focus on evaluating a new proposed approach based on LBP for texture classification in lung CT. In future work, we will evaluate the performance of this classification system for emphysema quantification. Another issue that would be interesting to investigate is to use the combination of LBP and filter responses, for example the filters described in Section 2.2 LBP3 and FB2 
achieve almost the same overall performance, but do not classify exactly the same ROIs correctly as indicated by the confusion matrices. Perhaps a combination of these features may further reduce classification errors.

To conclude, we have proposed to use local binary patterns combined with intensity for classification of texture in lung CT, achieving a classification accuracy of $95.2 \%$ on a three-class problem comprising normal tissue, centrilobular emphysema, and paraseptal emphysema.

Acknowledgements. This work is partly funded by the Danish Council for Strategic Research (NABIIT), the Netherlands Organisation for Scientific Research (NWO), and AstraZeneca, Lund, Sweden.

\section{References}

1. Rabe, et al.: Global strategy for the diagnosis, management, and prevention of chronic obstructive pulmonary disease: GOLD executive summary. Am. J. Respir. Crit. Care. Med. 176(6), 532-555 (2007)

2. Shaker, et al.: Identification of patients with chronic obstructive pulmonary disease (COPD) by measurement of plasma biomarkers. The Clinical Respiratory Journal 2(1), 17-25 (2008)

3. Müller, et al.: density mask, an objective method to quantitate emphysema using computed tomography. Chest 94(4), 782-787 (1988)

4. Webb, et al.: High-Resolution CT of the Lung, 3rd edn. Lippincott Williams \& Wilkins (2001)

5. Stavngaard, et al.: Quantitative assessment of regional emphysema distribution in patients with chronic obstructive pulmonary disease (COPD). Acta Radiol. 47(9), 914-921 (2006)

6. Tuceryan, M., Jain, A.K.: Texture analysis. In: The Handbook of Pattern Recognition and Computer Vision, 2nd edn., pp. 207-248. World Scientific Publishing, Singapore (1998)

7. Uppaluri, et al.: Quantification of pulmonary emphysema from lung computed tomography images. Am. J. Respir. Crit. Care. Med. 156(1), 248-254 (1997)

8. Chabat, et al.: Obstructive lung diseases: texture classification for differentiation at CT. Radiology 228(3), 871-877 (2003)

9. Xu, et al.: MDCT-based 3-D texture classification of emphysema and early smoking related lung pathologies. IEEE Trans. Med. Imaging 25(4), 464-475 (2006)

10. Sluimer, et al.: Computer-aided diagnosis in high resolution CT of the lungs. Med. Phys. 30(12), 3081-3090 (2003)

11. Ojala, et al.: Multiresolution gray-scale and rotation invariant texture classification with local binary patterns. IEEE Trans. Pattern Anal. Mach. Intell. 24(7), 971-987 (2002)

12. Oliver, et al.: False positive reduction in mammographic mass detection using local binary patterns. MICCAI 10(Pt. 1), 286-293 (2007)

13. Jain, et al.: Statistical pattern recognition: a review. IEEE Trans. Pattern Anal. Mach. Intell. 22(1), 4-37 (2000)

14. Rubner, et al.: A metric for distributions with applications to image databases. In: Sixth International Conference on Computer Vision, 1998, January 4-7, pp. 59-66 (1998)

15. Dietterich, T.G.: Approximate statistical test for comparing supervised classification learning algorithms. Neural Computation 10(7), 1895-1923 (1998) 